\title{
Robust Nonlinear Control of AC Brushless Motor for Electric Vehicles Application
}

\author{
Diego Langarica-Córdoba ${ }^{\dagger}$, Gerardo V. Guerrero-Ramírez*, Abraham Claudio-Sánchez*, \\ Miguel A. Durán-Fonseca*, Manuel Adam-Medina*, and Carlos-Manuel Astorga-Zaragoza* \\ ${ }^{\dagger *}$ Department of Electronic Engineering, Centro Nacional de Investigación y Desarrollo Tecnológico, Morelos, México
}

\begin{abstract}
This article proposes a robust nonlinear control based on Lyapunov's redesign, whose purpose is to deal with parametric uncertainty in the resistance of the motor windings. The robust controller design is based on the passivity properties of the motor, as well as energy shaping and damping injection. The application of this control technique is focused on electric vehicles mainly formed by a battery bank, a power inverter, an AC brushless motor and the mechanical transmission. The sine PWM technique is used to trigger the switching devices of inverter. The results were obtained from simulation, where is shown that robust control makes a proper tracking of electromagnetic torque.
\end{abstract}

Key Words: Electric vehicles, Lyapunov's stability, Passivity, Robust nonlinear control, Sine PWM

\section{INTRODUCTION}

The electric vehicle (EV) does not damage our environment because it does not emits gases. The global need for oil and strong emissions to the atmosphere by combustion cars makes electric transportation an effective solution. In recent years the development of the EV has increased, also the development of electric motors capable of satisfying the traction requirements. The AC brushless motor (ACBM) has significant advantages over other electric machines: there is no slip, maintance free because there is no slip rings, permanent magnet in the rotor requires no electricity to the field excitation, high ratio of power/weight, robustness and efficiency [3]. The ACBM is also used in power generation systems and as servo-systems actuator [12].

Several types of controllers were designed in order to achieve their control objectives under specific conditions. In [17], a sensorless control technique is proposed using and observer (easily implementable) for both angular position and speed of the PMSM rotor, The design of this observer is based on immersion and invariance technique. For example, [20] proposes a control technique to perform vector control of a Permanent Magnet Synchronous Motor (PMSM) without measurement of the rotor position and speed using high frequency voltage injection into stator terminals. Also, [1] proposes a sensorless control for a synchronous motor in a single piston rotary compressor, this technique is focused to reduce speed ripples, as well as, mechanical noise and

\footnotetext{
Manuscript received Dec. 30, 2010; revised Apr. 4, 2011

Recommended for publication by Guest Associate Editor Chris Edrington.

$\dagger$ Corresponding Author: d_langarica08e@ cenidet.edu.mx

Tel: +52-777-362-7770, CENIDET

* Department of Electronic Engineering, Centro Nacional de Investigación y Desarrollo Tecnológico, México
}

vibration. In [8] a robust adaptive backstepping controller is proposed for the speed control of PMSM in pure electrical vehicles applications under several electrical and mechanical parameters uncertanties. Another good reference in EV speed control is [7], this shows the road estimation and speed control of a car with four PMSM mounted in each wheel. The advantage of this configuration is that each wheel torque can be controlled completely and independently from the others. [2] and [14] are two good references of Passivity Based Control for nonlinear systems.

The next sections presents: three-phase and two-phase Euler-Lagrange (E-L) models for the ACBM, EV model, nominal control design based on passivity and robust control design based on Lyapunov's redesign to deal with parametric uncertainty in the resistance of ACBM windings. A controller is designed to achieve the torque tracking objective in all EV operation zone, ensuring signals bounding and internal stability of the entire system. Simulations are presented to illustrate the performance of this controller taking into account variations in electrical either mechanical parameters.

\section{System Model}

This section presents the mathematical model development proposed for the EV, which is usually formed by an electrical subsystem (battery bank, three-phase power inverter and motor) and a mechanical subsystem (motor, mechanical transmission and wheels), both illustrated in figure 1. The ACBM is part of both subsystems, because is responsible to transform the electric energy into mechanical energy and also provides the necessary traction force for EV motion. The battery model is considered an ideal voltage source capable of delivering the motor current demanded from it. 


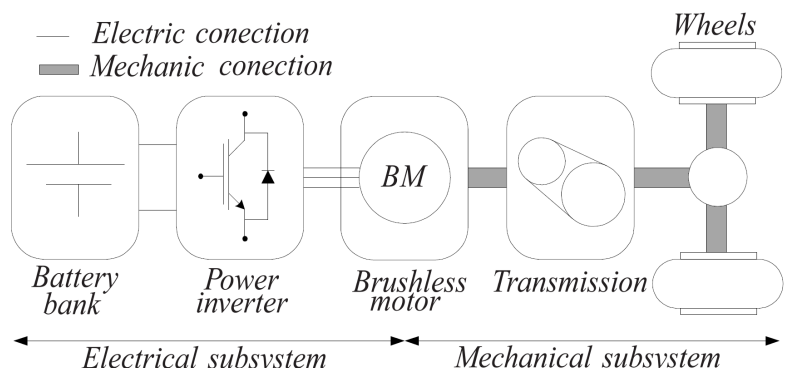

Fig. 1. EV diagram.

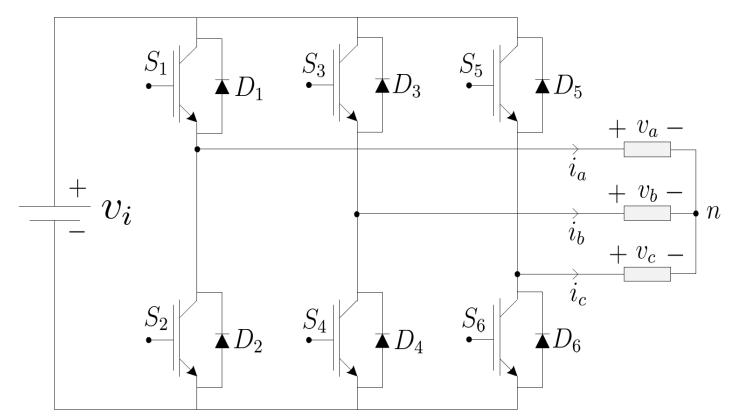

Fig. 2. Inverter Topology.

\section{A. Power Inverter Model Using Sine PWM Technique}

The DC-AC power electronic converter is also called inverter and the general purpose of this topology is to control amplitude, phase and frequency of the generated three-phase voltages. The presented model is reported in [19] and only considers ideal switching devices ( [15] and [18] presents an electro-thermal model for switching devices and a high frequency IGBT model, respectively). Fig. 2 shows the general inverter topology formed by six switching devices $\left(S_{1}, S_{2}\right.$, $S_{3}, S_{4}, S_{5}$ and $S_{6}$ ) and six free-wheeling diodes. The carrier signal used in sine PWM modulation is a triangular wave form represented by $g(t)=\frac{2}{\pi} \arcsin \left(\sin \left(2 \pi f_{s} t+\frac{\pi}{2}\right)\right)$, where $f_{s}$ is the carrier frequency [16]. Modulating signals are sine control signals $\left(M_{a}, M_{b}, M_{c}\right)$ and are also shown in figure 3.

Sine PWM technique compares the modulating signals with the carrier signal to produce binary trigger variables $a, b$ and $c$. For example, if at any time the modulating signal $M_{a}$ is bigger than carrier signal $g(t)$, then $a$ is a logic one so $S_{1}$ is on and $S_{2}$ is off. In the case that $M_{a}$ is lower than $g(t)$, then $a$ is a logic zero so $S_{1}$ is off and $S_{2}$ is on. The equation (1) relates the binary variables $a, b$ and $c$ with voltage $v_{i}$ to generate the phase voltages $v_{a}, v_{b}$ and $v_{c}$, where $v_{i}$ represents the output voltage of the battery bank.

$$
\left[\begin{array}{l}
v_{a} \\
v_{b} \\
v_{c}
\end{array}\right]=\frac{v_{i}}{3}\left[\begin{array}{ccc}
2 & -1 & -1 \\
-1 & 2 & -1 \\
-1 & -1 & 2
\end{array}\right]\left[\begin{array}{l}
a \\
b \\
c
\end{array}\right]
$$

For the case of close-loop system operation, three-phase signals from nonlinear controller are divided by the voltage bus $v_{i}$ to produce modulating signals. [10] is a good reference that proposes a passivity-based control strategy for single-phase inverters, this approach is an alternative to ours.

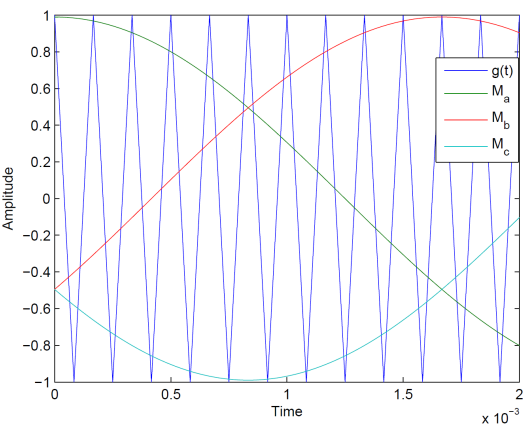

Fig. 3. Carrier and modulating signals.

\section{B. AC Brushless Motor Model in Euler-Lagrange Formulation}

The AC BM is a Permanent Magnet Synchronous Motor in which the rotor angular velocity is related to the angular velocity of the rotating magnetic field in the stator and there is no slip between this two velocities. The stator contains multiple coils per phase winding distributed in slots around it. The rotor consists of one or more permanent magnets with the aim of generating the magnetic field of the rotor. For our analysis it is assumed that the stator windings are sinusoidally distributed. The theory to obtain the model is available at [13]. The E-L equations for both, electrical and mechanical subsystems are described by:

$$
\begin{aligned}
\Sigma_{e}: & D_{e} \ddot{q}_{e}+W_{1} \dot{q}_{m} \dot{q}_{e}+W_{2} \dot{q}_{m}+R_{e} \dot{q}_{e}=M_{e} u \text { and } \\
\Sigma_{m}: & D_{M B} \ddot{q}_{m}-\tau_{e m}+R_{m} \dot{q}_{m}=-\tau_{L} .
\end{aligned}
$$

The electromagnetic torque is defined as:

$$
\tau_{e m}=\frac{1}{2} \dot{q}_{e}^{T} W_{1} \dot{q}_{e}+\dot{q}_{e}^{T} W_{2} .
$$

For electrical subsystem $\Sigma_{e}$ the generalized coordinates are defined as $q_{e}=\left[\begin{array}{lll}q_{1} & q_{2} & q_{3}\end{array}\right]^{\top}$, which represents the electric charges in motor windings. The generalized velocities $\dot{q}_{e}=\left[\begin{array}{lll}\dot{q}_{1} & \dot{q}_{2} & \dot{q}_{3}\end{array}\right]^{\top}=\left[\begin{array}{lll}i_{a} & i_{b} & i_{c}\end{array}\right]^{\top}$ are the currents in the motor windings. For mechanical subsystem $\Sigma_{m}$ the generalized coordinate is defined as the mechanical angular position $q_{m}$. The mechanical angular velocity is $\dot{q}_{m} . R_{e}=\operatorname{diag}\left\{r_{e}, r_{e}, r_{e}\right\}$ is the resistance matrix of the stator windings. $M_{e}$ is a $3 \times 3$ dimensions identity matrix and the control input is $u=\left[\begin{array}{lll}u_{1} & u_{2} & u_{3}\end{array}\right]^{\top}$, corresponding to the stator voltages. $D_{M B}, R_{m}$ and $\tau_{L}$ are the ACBM inertia, the viscous friction coefficient and load torque respectively. The elements of the inductance matrix $D_{e}$ are defined in [11] by

$$
D_{e}=\left[\begin{array}{lll}
l_{a a} & l_{a b} & l_{a c} \\
l_{b a} & l_{b b} & l_{b c} \\
l_{c a} & l_{c b} & l_{c c}
\end{array}\right],
$$

where each element is represented by:

$$
\begin{aligned}
& l_{a a}=L_{l s}+\bar{L}_{m}-L_{\Delta m} \cos 2\left(n_{p} \theta_{m}\right) \\
& l_{b b}=L_{l s}+\bar{L}_{m}-L_{\Delta m} \cos 2\left(n_{p} \theta_{m}-2 \pi / 3\right) \\
& l_{c c}=L_{l s}+\bar{L}_{m}-L_{\Delta m} \cos 2\left(n_{p} \theta_{m}+2 \pi / 3\right) \\
& l_{a b}=l_{b a}=-\bar{L}_{m} / 2-L_{\Delta m} \cos 2\left(n_{p} \theta_{m}-\pi / 3\right) \\
& l_{a c}=l_{c a}=-\bar{L}_{m} / 2-L_{\Delta m} \cos 2\left(n_{p} \theta_{m}+\pi / 3\right) \\
& l_{b c}=l_{c b}=-\bar{L}_{m} / 2-L_{\Delta m} \cos 2\left(n_{p} \theta_{m}+\pi\right) .
\end{aligned}
$$


$L_{l s}$ is the leakage inductance, $\bar{L}_{m}$ is the average value of magnetizing inductance and $L_{\Delta m}$ is the peak value of magnetizing inductance. $W_{1}=\partial D_{e} / \partial q_{m}$ and $W_{2}=\partial \mu / \partial q_{m}$, where $\mu$ is the flux linkages vector due to the permanent magnets and is represented as:

$$
\mu=\lambda_{m}\left[\begin{array}{c}
\sin \left(n_{p} q_{m}\right) \\
\sin \left(n_{p} q_{m}-2 \pi / 3\right) \\
\sin \left(n_{p} q_{m}+2 \pi / 3\right)
\end{array}\right] .
$$

$\lambda_{m}$ is the flux linkages amplitude and is determined by the magnetic material in the rotor, $n_{p}$ is the pole pair number of the motor. The angular electric position $\theta_{e}$ is related with the angular mechanic position $q_{m}$ by $\theta_{e}=n_{p} q_{m}$.

\section{Motor Model in Fixed Rotor Reference Frame}

The mathematical model of the electrical subsystem $\Sigma_{e}$ given by the equation (2), has coefficients which depend on the rotor position. The Park's transformation is used to eliminate this dependence. This transformation allows refer all electric variables to the fixed rotor reference frame using $f_{q d 0}=k_{s}^{r} f_{a b c}$ where the Park's transformation matrix $k_{s}^{r}$ is given by [12]:

$$
k_{s}^{r}=\frac{2}{3}\left[\begin{array}{ccc}
\cos n_{p} q_{m} & \cos \left(n_{p} q_{m}-\frac{2 \pi}{3}\right) & \cos \left(n_{p} q_{m}+\frac{2 \pi}{3}\right) \\
\sin n_{p} q_{m} & \sin \left(n_{p} q_{m}-\frac{2 \pi}{3}\right) & \sin \left(n_{p} q_{m}+\frac{2 \pi}{3}\right) \\
1 / 2 & 1 / 2 & 1 / 2
\end{array}\right]
$$

This transformation is only used for the electrical subsystem in equation (2), this equation now is expressed in function of $(q d 0)$ variables, in other words, $\ddot{q}_{e}=\left(k_{s}^{r}\right)^{-1} \ddot{q}_{e}^{r}, D_{e}=$ $\left(k_{s}^{r}\right)^{-1} D_{e}^{r}, \mu=\left(k_{s}^{r}\right)^{-1} \mu^{r}, \dot{q}_{e}=\left(k_{s}^{r}\right)^{-1} \dot{q}_{e}^{r}$ and $u=\left(k_{s}^{r}\right)^{-1} u^{r}:$

$$
\begin{array}{r}
D_{e}\left(k_{s}^{r}\right)^{-1} \ddot{q}_{e}^{r}+\frac{\partial\left(k_{s}^{r}\right)^{-1} D_{e}^{r}}{\partial q_{m}} \dot{q}_{m} \dot{q}_{e}+\frac{\partial\left(k_{s}^{r}\right)^{-1} \mu^{r}}{\partial q_{m}} \dot{q}_{m} \ldots \\
\ldots+R_{e}\left(k_{s}^{r}\right)^{-1} \dot{q}_{e}^{r}=M_{e}\left(k_{s}^{r}\right)^{-1} u^{r} .
\end{array}
$$

Now all terms are multiplied by $k_{s}^{r}$ :

$$
\begin{gathered}
k_{s}^{r} D_{e}\left(k_{s}^{r}\right)^{-1} \ddot{q}_{e}^{r}+k_{s}^{r} \frac{\partial\left(k_{s}^{r}\right)^{-1} D_{e}^{r}}{\partial q_{m}} \dot{q}_{m} \dot{q}_{e}+k_{s}^{r} \frac{\partial\left(k_{s}^{r}\right)^{-1} \mu^{r}}{\partial q_{m}} \dot{q}_{m} \ldots \\
\ldots+k_{s}^{r} R_{e}\left(k_{s}^{r}\right)^{-1} \dot{q}_{e}^{r}=k_{s}^{r} M_{e}\left(k_{s}^{r}\right)^{-1} u^{r} .
\end{gathered}
$$

If we consider that $\mu^{r}=k_{s}^{r} \mu\left(q_{m}\right)=\left[\begin{array}{lll}0 & \lambda_{m} & 0\end{array}\right]^{\top}$ and developing all the indicated operations in equation (9) then the model for the electrical subsystem $\Sigma_{e}$ in fixed rotor reference frame is:

$$
D_{e}^{r} \ddot{q}_{e}^{r}+W_{1}^{r} \dot{q}_{m} \dot{q}_{e}^{r}+W_{2}^{r} \dot{q}_{m}+R_{e}^{r} \dot{q}_{e}^{r}=u^{r},
$$

with

$$
\begin{gathered}
D_{e}^{r}=\left[\begin{array}{ccc}
L_{q} & 0 & 0 \\
0 & L_{d} & 0 \\
0 & 0 & L_{l s}
\end{array}\right], W_{1}^{r}=n_{p}\left[\begin{array}{ccc}
0 & L_{d} & 0 \\
-L_{q} & 0 & 0 \\
0 & 0 & 0
\end{array}\right], \\
W_{2}^{r}=\left[\begin{array}{c}
n_{p} \lambda_{m} \\
0 \\
0
\end{array}\right] \text { and } R_{e}^{r}=\left[\begin{array}{ccc}
r_{e} & 0 & 0 \\
0 & r_{e} & 0 \\
0 & 0 & 0
\end{array}\right] .
\end{gathered}
$$

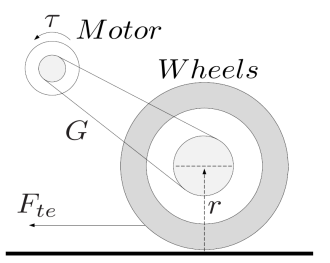

Fig. 4. EV transmission.

The control signal is now defined as $u^{r}=\left[\begin{array}{lll}u_{1}^{r} & u_{2}^{r} & u_{3}^{r}\end{array}\right]^{\top}=$ $\left[\begin{array}{lll}v_{q} & v_{d} & v_{0}\end{array}\right]^{\top}$ and current vector as $\dot{q}_{e}^{r}=\left[\begin{array}{lll}\dot{q}_{1}^{r} & \dot{q}_{2}^{r} & \dot{q}_{3}^{r}\end{array}\right]^{\top}=$ $\left[\begin{array}{lll}i_{q} & i_{d} & i_{0}\end{array}\right]^{\top}$. The new expression of electromagnetic torque is:

$$
\tau_{e m}=\frac{3}{2} n_{p} \dot{q}_{1}^{r}\left(\left(L_{d}-L_{q}\right) \dot{q}_{2}^{r}+\lambda_{m}\right) .
$$

The $q$ and $d$ inductances are: $L_{q}=L_{l s}+\frac{3}{2} \bar{L}_{m}-\frac{3}{2} L_{\Delta m}$ and $L_{d}=L_{l s}+\frac{3}{2} \bar{L}_{m}+\frac{3}{2} L_{\Delta m}$.

When stator windings resistances have the same value, the resistance matrix of the stator is:

$$
R_{e}=\left[\begin{array}{ccc}
r_{e} & 0 & 0 \\
0 & r_{e} & 0 \\
0 & 0 & r_{e}
\end{array}\right]
$$

and satisfies

$$
R_{e}^{r}=k_{s}^{r} R_{e}\left(k_{s}^{r}\right)^{-1}=R_{e},
$$

in other words, despite the transformation, the resistance matrix remains exactly as before to apply this transformation. In the other hand, when stator windings resistances have different value, the resistance matrix of the stator is represented by single phase resistance value:

$$
R_{e}=\left[\begin{array}{ccc}
r_{a} & 0 & 0 \\
0 & r_{b} & 0 \\
0 & 0 & r_{c}
\end{array}\right]
$$

and the transformation to fixed rotor reference frame is:

$$
R_{e x}^{r}=k_{s}^{r} R_{e x}\left(k_{s}^{r}\right)^{-1}=\left[\begin{array}{lll}
r_{11} & r_{12} & r_{13} \\
r_{21} & r_{22} & r_{23} \\
r_{31} & r_{32} & r_{33}
\end{array}\right]
$$

where $r_{11}=\frac{2}{3} r_{a}+\frac{1}{6} r_{b}+\frac{1}{6} r_{c}, r_{12}=\frac{\sqrt{3}}{6}\left(r_{b}-r_{c}\right), r_{13}=$ $\frac{2}{3} r_{a}-\frac{1}{3} r_{b}-\frac{1}{3} r_{c}, r_{21}=\frac{1}{6}\left(r_{b}-r_{c}\right), r_{22}=\frac{1}{2}\left(r_{b}+r_{c}\right), r_{23}=$ $\frac{\sqrt{3}}{3}\left(r_{b}-r_{c}\right), r_{31}=\frac{1}{3} r_{a}-\frac{1}{6} r_{b}-\frac{1}{6} r_{c}, r_{32}=-\frac{\sqrt{3}}{6}\left(r_{b}-r_{c}\right)$. $r_{33}=\frac{1}{3}\left(r_{a}+r_{b}+r_{c}\right)$.

The subscript $R_{e x}$ refers to an explicit resistances matrix and this is used in the robust nonlinear controller design.

\section{Electric Vehicle Model}

When a simple transmission like in figure 4 is considered [5] [6], the equation used to modelling this transmission is:

$$
\tau=\frac{r}{\eta_{g} G} F_{t e}
$$

Where $r$ is the radius of the wheel, $\eta_{g}$ is the trasmission efficiency, $G$ is the reduction ratio of the angular speed of the transmission, $\tau$ is the torque given in the motor shaft and $F_{t e}$ is the traction force which makes possible the EV motion. In figure 4 we obtain the relationship between angular speed 


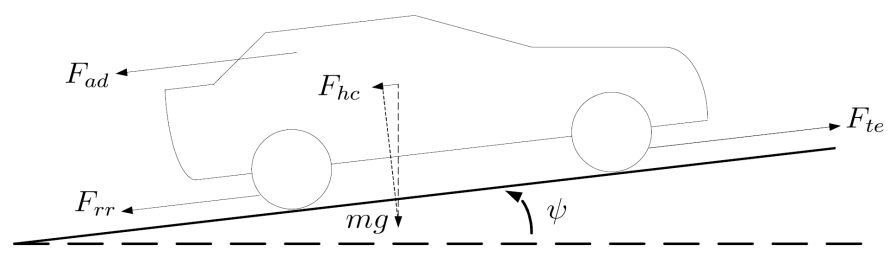

Fig. 5. Forces acting on the car.

$\dot{q}_{m}$ and lineal speed $v$ of the EV with:

$$
\dot{q}_{m}=G \frac{v}{r} \text {. }
$$

The forces acting on the car are shown in figure 5. The force $F_{t e}$ drives the vehicle and is provided by the electric motor through the transmission. The friction force $F_{r r}$ between the wheels and ground is given by $F_{r r}=\mu_{r r} m g \cos (\psi)$, where $\mu_{r r}$ is a friction coefficient, $m$ is the total mass of the EV in $K g, g=9.8 \mathrm{~m} / \mathrm{s}^{2}$ is the gravity constant and $\psi$ is the inclination angle.

The force given by the air is $F_{a d}=\frac{1}{2} \rho A C_{d} v^{2}$, where $\rho=$ $1.25 \mathrm{~kg} / \mathrm{m}^{3}$ is the air density, $A$ is the frontal area of the car in $m^{2}, C_{d}$ is the aerodynamic coefficient and $v$ is the lineal speed in $\mathrm{m} / \mathrm{s}$. The force $F_{h c}$ is a function of inclination angle $\psi$ and is represented by $F_{h c}=m g \sin (\psi)$.

With the Newton's second law we obtain:

$$
F_{t e}-F_{r r}-F_{a d}-F_{h c}=m a,
$$

where $a$ is the car aceleration in $m / s^{2}$. Solving for the traction force $F_{t e}$ in equation (18) and considering all expressions of the forces acting on the car, the next expression is obtained:

$$
F_{t e}=m a+\mu_{r r} m g \cos (\psi)+\frac{1}{2} \rho A C_{d} v^{2}+m g \sin (\psi)
$$

In this last equation (19), the $\mathrm{AC}$ brushless motor and the transmission have not been considered. In the mechanical subsystem $\Sigma_{m}$ given by equation (2), the electromagnetic torque is:

$$
\tau_{e m}=D_{M B} \ddot{q}_{m}+R_{m} \dot{q}_{m}+\tau_{L} .
$$

The total inertia is the sum of the BM inertia and the EV inertia:

$$
D_{m}=D_{M B}+D_{V E} .
$$

The inertia of the $\mathrm{EV}$ is $D_{V E}=\frac{1}{2} m \frac{r^{2}}{G^{2}}$. The equations (16) and (21) are replaced in (20) and the next expression is obtained:

$$
\tau_{e m}=D_{m} \ddot{q}_{m}+R_{m} \dot{q}_{m}+\frac{r}{\eta_{g} G} F_{t e}
$$

When the equation (19) is replaced in (22), results:

$$
\begin{array}{r}
\tau_{e m}=D_{m} \ddot{q}_{m}+R_{m} \dot{q}_{m}+\frac{r}{\eta_{g} G}\left(m \frac{d v}{d t}+\mu_{r r} m g \cos (\psi) \ldots\right. \\
\left.\ldots+\frac{1}{2} \rho A C_{d} v^{2}+m g \sin (\psi)\right) .
\end{array}
$$

The equation (23) contains both angular speed $\dot{q}_{m}$ and lineal speed $v$. Finally the mechanical subsystem model is obtained when the equation (23) is only in function of $\dot{q}_{m}$ using equation (17) and solving for $\ddot{q}_{m}$ :

$$
\begin{array}{r}
\ddot{q}_{m}=\frac{\eta_{g} G^{2}}{D_{m} \eta_{g} G^{2}+m r^{2}}\left(\tau_{e m}-R_{m} \dot{q}_{m}-\frac{\mu_{r r} m g r \cos (\psi)}{\eta_{g} G} \ldots\right. \\
\left.\ldots-\frac{\rho A C_{d} r^{3}}{2 \eta_{g} G^{3}} \dot{q}_{m}^{2}-\frac{m g r \sin (\psi)}{\eta_{g} G}\right) .
\end{array}
$$

This equation (24) represents all the mechanical subsystem of the EV given by figure 1, the mechanical dynamics of the BM is also included.

\section{Robust Controller Design}

\section{A. Nominal Control Design Based on Passivity}

The nominal control design for the ACBM is divided in two parts: a controller for mechanical subsystem and a controller for electrical subsystem. For the mechanical subsystem the controller design begin with equation (20) and its desired dynamics:

$$
\begin{aligned}
D_{m} \ddot{q}_{m}+R_{m} \dot{q}_{m}+\tau_{L} & =\tau_{e m} \text { and } \\
D_{m} \ddot{q}_{m d}+R_{m} \dot{q}_{m d}+\tau_{L} & =\tau_{e m d} .
\end{aligned}
$$

The subscript ${ }_{d}$ refer to the desired trajectories. Now the speed and acceleration errors are defined as $\dot{\tilde{q}}_{m}=\dot{q}_{m}-\dot{q}_{m d}$ and $\ddot{\tilde{q}}_{m}=\ddot{q}_{m}-\ddot{q}_{m d}$ respectively. The error dynamic equation is obtained by subtract equations in (25):

$$
D_{m} \ddot{\tilde{q}}_{m}+R_{m} \dot{\tilde{q}}_{m}=\tau_{e m}-\left[D_{m} \ddot{q}_{m d}+R_{m} \dot{q}_{m d}+\tau_{L}\right] .
$$

The second member of the last equation is the input to the error dynamic equation and is defined as:

$$
\sigma_{m}=\tau_{e m}-\left[D_{m} \ddot{q}_{m d}+R_{m} \dot{q}_{m d}+\tau_{L}\right],
$$

hence, if $\tau_{e m}=\tau_{e m d}$, then, $\sigma_{m}=0$. The next Lyapunov's function is proposed to design the controller for the mechanical subsystem:

$$
V\left(\dot{\tilde{q}}_{m}\right)=\frac{1}{2} D_{m} \dot{\tilde{q}}_{m}^{2},
$$

as $D_{m} \geq 0$ and also $\dot{\tilde{q}}^{2}>0$, it can say that $V\left(\dot{\tilde{q}}_{m}\right)>0$, also, $V(0)=0, V\left(\dot{\tilde{q}}_{m}\right) \neq 0$ and $V(\infty) \rightarrow \infty$.

The derivative of the candidate function is:

$$
\dot{V}=\dot{\tilde{q}}_{m} D_{m} \ddot{\tilde{q}}_{m} .
$$

This derivative must be negative definite to ensure stability. The $D_{m} \ddot{\tilde{q}}_{m}$ term in equation (26) is replaced in equation (29), so we obtain:

$$
\dot{V}=\dot{\tilde{q}}_{m}\left(\tau_{e m}-\left[D_{m} \ddot{q}_{m d}+R_{m} \dot{q}_{m}+\tau_{L}\right]\right) .
$$

To force $\dot{V}$ be a defined negative function is considered:

$$
\tau_{e m}-\left[D_{m} \ddot{q}_{m d}+R_{m} \dot{q}_{m}+\tau_{L}\right]=-\Gamma_{m} \dot{\tilde{q}}_{m}, \Gamma_{m}>0 .
$$

So, $\dot{V}$ is now negative definite and its expression takes de form:

$$
\dot{V}=-\dot{\tilde{q}}_{m} \Gamma_{m} \dot{\tilde{q}}_{m}=-\Gamma_{m} \dot{\tilde{q}}_{m}^{2} .
$$


The required torque for reference speed tracking comes from $\tau_{e m}$ in equation (31). This torque and its derivative are renamed as $\tau_{\text {emd }}$ and $\dot{\tau}_{\text {emd }}$ and are defined by:

$$
\begin{aligned}
& \tau_{e m d}=-\Gamma_{m} \dot{\tilde{q}}_{m}+D_{m} \ddot{q}_{m d}+R_{m} \dot{q}_{m}+\tau_{L} \text { and } \\
& \dot{\tau}_{e m d}=-\Gamma_{m} \ddot{\tilde{q}}_{m}+D_{m} \dddot{q}_{m d}+R_{m} \ddot{q}_{m}+\dot{\tau}_{L} .
\end{aligned}
$$

The load torque and its derivative are defined in the EV model as:

$$
\begin{array}{r}
\tau_{L}=\frac{r}{\eta_{g} G}\left(\frac{m r}{G} \ddot{q}_{m}+\mu_{r r} m g \cos (\psi)+\frac{\rho A C_{d} r^{2}}{2 G^{2}} \dot{q}_{m}^{2} \ldots\right. \\
\ldots+m g \sin (\psi)), \\
\dot{\tau}_{L}=\frac{r}{\eta_{g} G}\left(\frac{m r}{G} \dddot{q}_{m}-\mu_{r r} m g \dot{\psi} \sin (\psi)+\frac{\rho A C_{d} r^{2}}{G^{2}} \dot{q}_{m} \ddot{q}_{m} \ldots\right. \\
\ldots+m g \dot{\psi} \cos (\psi)) .
\end{array}
$$

It is important to say that the nominal controller design for the electrical subsystem of the motor takes into account that $\dot{q}_{3}^{r}=i_{0}$ has a non-zero dynamic, because the windings resistances are unbalanced, they have not the same value. Also, the control signal has three elements, $u^{r}=\left[\begin{array}{lll}u_{1}^{r} & u_{2}^{r} & u_{3}^{r}\end{array}\right]^{\top}=$ $\left[\begin{array}{lll}v_{q} & v_{d} & v_{0}\end{array}\right]^{\top}$. In the controller design, the input trajectories are defined by the desired torque and its derivative in equation (33). The desired currents are obtained from the equation:

$$
\tau_{e m d}=\frac{3}{2} n_{p} \dot{q}_{1 d}^{r}\left(\left(L_{d}-L_{q}\right) \dot{q}_{2 d}^{r}+\lambda_{m}\right)
$$

To emulate the torque in a DC motor, is considered $\dot{q}_{2 d}^{r}=0$, then the last expression takes the form:

$$
\tau_{e m d}=\frac{3}{2} n_{p} \lambda_{m} \dot{q}_{1 d}^{r}
$$

also it is desired that the zero phase current be $\dot{q}_{3 d}^{r}=0$. Solving for $\dot{q}_{1 d}^{r}$ in the last equation, the desired current vector and its derivative is:

$$
\dot{q}_{e d}^{r}=\left[\begin{array}{c}
\frac{2 \tau_{e m d}}{3 n_{p} \lambda_{m}} \\
0 \\
0
\end{array}\right] \text { and } \ddot{q}_{e d}^{r}=\left[\begin{array}{c}
\frac{2 \dot{\tau}_{e m d}}{3 n_{p} \lambda_{m}} \\
0 \\
0
\end{array}\right] .
$$

Now, the equations for the electrical subsystem and its desired dynamics are:

$$
\begin{aligned}
D_{e}^{r} \ddot{q}_{e}^{r}+W_{1}^{r} \dot{q}_{m} \dot{q}_{e}^{r}+W_{2}^{r} \dot{q}_{m}+R_{e}^{r} \dot{q}_{e}^{r} & =u^{r} \text { and } \\
D_{e}^{r} \ddot{q}_{e d}^{r}+W_{1}^{r} \dot{q}_{m} \dot{q}_{e d}^{r}+W_{2}^{r} \dot{q}_{m}+R_{e}^{r} \dot{q}_{e d}^{r} & =u_{d}^{r} .
\end{aligned}
$$

The electric error and its derivative are defined as:

$$
\begin{gathered}
e=\dot{q}_{e}^{r}-\dot{q}_{e d}^{r}=\left[\begin{array}{c}
\dot{q}_{1}^{r} \\
\dot{q}_{2}^{r} \\
\dot{q}_{3}^{r}
\end{array}\right]-\left[\begin{array}{c}
\dot{q}_{1 d}^{r} \\
\dot{q}_{2 d}^{r} \\
\dot{q}_{3 d}^{r}
\end{array}\right] \text { and } \\
\dot{e}=\ddot{q}_{e}^{r}-\ddot{q}_{e d}^{r}=\left[\begin{array}{c}
\ddot{q}_{1}^{r} \\
\ddot{q}_{2}^{r} \\
\ddot{q}_{3}^{r}
\end{array}\right]-\left[\begin{array}{c}
\ddot{q}_{1 d}^{r} \\
\ddot{q}_{2 d}^{r} \\
\ddot{q}_{3 d}^{r}
\end{array}\right] .
\end{gathered}
$$

Substracting the systems dynamics and the desired dynamics, the electric error dynamic equation is obtained:

$$
D_{e}^{r} \dot{e}+W_{1}^{r} \dot{q}_{m} e+R_{e}^{r} e=\sigma_{e},
$$

with the input defined as:

$$
\sigma_{e}=u^{r}-\left[D_{e}^{r} \ddot{q}_{e d}^{r}+W_{1}^{r} \dot{q}_{m} \dot{q}_{e d}^{r}+W_{2}^{r} \dot{q}_{m}+R_{e}^{r} \dot{q}_{e d}^{r}\right] .
$$

For design purposes, the resulting term in $W_{1}^{r} \dot{q}_{m}$ requires acomplish with skew-symmetrical property, that is to say, a transposed matrix is equal than the original matrix $(A=$ $-A^{\top}$ ). To acomplish this property, the matrix $Z_{1}$ is proposed as:

$$
Z_{1}=\left[\begin{array}{ccc}
0 & \left(L_{d}-L_{q}\right) n_{p} \dot{q}_{m} & 0 \\
0 & 0 & 0 \\
0 & 0 & 0
\end{array}\right]
$$

Adding and subtracting $Z_{1}$ to the error dynamic equation, results:

$$
D_{e}^{r} \dot{e}+\left[W_{1}^{r} \dot{q}_{m}-Z_{1}\right] e+\left[R_{e}^{r}+Z_{1}\right] e=\sigma_{e} .
$$

Then:

$$
\begin{gathered}
C_{e}^{r}=W_{1}^{r} \dot{q}_{m}-Z_{1}=n_{p} \dot{q}_{m}\left[\begin{array}{ccc}
0 & L_{q} & 0 \\
-L_{q} & 0 & 0 \\
0 & 0 & 0
\end{array}\right] \text { and } \\
\bar{R}_{e}^{r}=R_{e}^{r}+Z_{1}=n_{p}\left[\begin{array}{ccc}
r_{e} & \left(L_{d}-L_{q}\right) n_{p} \dot{q}_{m} & 0 \\
o & r_{e} & 0 \\
0 & 0 & r_{e}
\end{array}\right],
\end{gathered}
$$

where $C_{e}^{r}$ acomplish with skew-symmetry property. Under this new transformation, the dynamic error equation is:

$$
D_{e}^{r} \dot{e}+C_{e}^{r} e+\bar{R}_{e}^{r} e=\sigma_{e},
$$

where $\sigma_{e}=u^{r}-\left[D_{e}^{r} \ddot{q}_{e d}^{r}+C_{e}^{r} \dot{q}_{e d}^{r}+W_{2}^{r} \dot{q}_{m}+\bar{R}_{e}^{r} \dot{q}_{e d}^{r}\right]$.

If $u^{r}=u_{d}^{r}$, then $\sigma_{e}=0$, therefore the control signal in closeloop is:

$$
u^{r}=D_{e}^{r} \ddot{q}_{e d}^{r}+C_{e}^{r} \dot{q}_{e d}^{r}+W_{2}^{r} \dot{q}_{m}+\bar{R}_{e}^{r} \dot{q}_{e d}^{r} .
$$

To get a fast convergence, damping is added to the dynamic error equation, the product between a gain matrix $K_{1}$ and the current error $e$ is included in both members of the equation:

$$
D_{e}^{r} \dot{e}+C_{e}^{r} e+\bar{R}_{e}^{r} e+K_{1} e=\sigma_{e}+K_{1} e .
$$

Under this damping injection, the nominal control law is:

$$
u^{r}=D_{e}^{r} \ddot{q}_{e d}^{r}+C_{e}^{r} \dot{q}_{e d}^{r}+W_{2}^{r} \dot{q}_{m}+\bar{R}_{e}^{r} \dot{q}_{e d}^{r}-K_{1} e .
$$

The Lyapunov's stability analysis is used to find all elements of $K_{1}$, the proposed candidate function is:

$$
V=\frac{1}{2} e^{\top} D_{e}^{r} e .
$$

$V$ is positive definite because $D_{e}^{r}>0$ and there is a cuadratic product in error. The derivative of the candidate function is:

$$
\dot{V}=e^{\top} D_{e}^{r} \dot{e}
$$

From equation (47), when the input $\sigma_{e}+K_{1} e=0$, the $D_{e}^{r} \dot{e}$ term is:

$$
D_{e}^{r} \dot{e}=-C_{e}^{r} e-\left[\bar{R}_{e}^{r}+K_{1}\right] e .
$$

Then, replacing $D_{e}^{r} \dot{e}$ in (50), results:

$$
\dot{V}=-e^{\top} C_{e}^{r} e-e^{\top}\left[\bar{R}_{e}^{r}+K_{1}\right] e=-e^{\top}\left[\bar{R}_{e}^{r}+K_{1}\right] e,
$$

where $e^{\top} C_{e}^{r} e=0$ because $C_{e}^{r}$ is skew-symmetrical. $\dot{V}$ must forced to be a negative definite function, then $\left[\bar{R}_{e}^{r}+K_{1}\right]$ must be a positive definite. Therefore, $K_{1}$ is proposed as:

$$
K_{1}=\left[\begin{array}{ccc}
k & -\left(L_{d}-L_{q}\right) n_{p} \dot{q}_{m} & 0 \\
0 & k & 0 \\
0 & 0 & k
\end{array}\right]
$$


hence

$$
\bar{R}_{e}^{r}+K_{1}=\left[\begin{array}{ccc}
r_{e}+k & 0 & 0 \\
0 & r_{e}+k & 0 \\
0 & 0 & r_{e}+k
\end{array}\right], \quad k>0 .
$$

This ensures that $\dot{V}<0$.

\section{B. Robust Control Design Based on Lyapunov's Redesign}

Robust control is designed to deal with parametric uncertainty in the resistance of the stator windings [4]. The resistance can change due a lot of factor like temperature, age or inter-turn short-circuits. To cope with the efect of the resistance change, an aditional term is designed and added to the nominal control law given in (48). To find this new term, we need the desired dynamic equation:

$$
u_{d}^{r}=D_{e}^{r} \ddot{q}_{e d}^{r}+C_{e}^{r} \dot{q}_{e d}^{r}+W_{2}^{r} \dot{q}_{m}+\bar{R}_{e x}^{r} \dot{q}_{e d}^{r},
$$

where

$$
\bar{R}_{e x}^{r}=R_{e x}^{r}+Z_{1}=\left[\begin{array}{ccc}
r_{11} & r_{12}+\left(L_{d}-L_{q}\right) n_{p} \dot{q}_{m} & r_{13} \\
r_{21} & r_{22} & r_{23} \\
r_{31} & r_{32} & r_{33}
\end{array}\right] .
$$

Taking into account (37) and developing all terms in (55), the desired dynamic equation can be grouped together into:

$$
\begin{aligned}
u_{d}^{r 0}= & {\left[\begin{array}{c}
\frac{L_{q} 2 \dot{\tau}_{e m d}}{3 n_{p} \lambda_{m}}+\lambda_{m} n_{p} \dot{q}_{m} \\
\frac{-\dot{q}_{m} L_{q} 2 \tau_{e m d}}{3 \lambda_{m}} \\
0
\end{array}\right]+} \\
& {\left[\begin{array}{rrr}
\frac{2}{3} \alpha & \frac{1}{6} \alpha & \frac{1}{6} \alpha \\
0 & \frac{1}{6} \alpha & -\frac{1}{6} \alpha \\
\frac{1}{3} \alpha & -\frac{1}{6} \alpha & -\frac{1}{6} \alpha
\end{array}\right]\left[\begin{array}{c}
r_{a}^{0} \\
r_{b}^{0} \\
r_{c}^{0}
\end{array}\right]=g_{d}^{0}+B_{d}^{0} \Phi^{0}, }
\end{aligned}
$$

where

$$
\alpha=\frac{2 \tau_{\text {emd }}}{3 n_{p} \lambda_{m}},
$$

and $g_{d}^{0}$ is the vector that contains the independent terms to $r_{e}$ without uncertainty, $B_{d}^{0}$ is a kind of regressor and contains terms that multiply $r_{e}, \Phi^{0}$ contains the parameter with uncertainty. The superscript ${ }^{0}$ indicates nominal value. The term $B_{d}^{0} w$ is added to the nominal control law to compensate for the parameter uncertainty.:

$$
u^{r}=u_{d}^{r 0}-K_{1} e+B_{d}^{0} w .
$$

The parametric error is defined as the difference between the nominal value and the actual value of $r_{e}$, that is to say,

$$
\tilde{\Phi}=\Phi^{0}-\Phi=\left[\begin{array}{c}
r_{a}^{0} \\
r_{b}^{0} \\
r_{c}^{0}
\end{array}\right]-\left[\begin{array}{l}
r_{a} \\
r_{b} \\
r_{c}
\end{array}\right]
$$

and it must satisfy

$$
\|\tilde{\Phi}\| \leq \rho_{1}, \quad \rho_{1} \in \mathbb{R}^{+} .
$$

$\rho_{1}$ is the maximum bound of the parameter error vector $\|\tilde{\Phi}\|$. With the Lyapunov's stability analisys $w$ can be found, for this, $\pm B_{d}^{0} \Phi$ is added to the equation (59) as:

$$
\begin{aligned}
u^{r} & =g_{d}^{0}+B_{d}^{0} \Phi^{0}-K_{1} e+B_{d}^{0} w \pm B_{d}^{0} \Phi \\
u^{r} & =\left(g_{d}^{0}+B_{d}^{0} \Phi\right)+B_{d}^{0}(\tilde{\Phi}+w)-K_{1} e \\
u^{r}-\left(g_{d}^{0}+B_{d}^{0} \Phi\right) & =B_{d}^{0}(\tilde{\Phi}+w)-K_{1} e .
\end{aligned}
$$

The term $u^{r}-\left(g_{d}^{0}+B_{d}^{0} \Phi\right)$ is the difference between real and desired behaviour, therefore, using (45):

$$
D_{e}^{r} \dot{e}+C_{e}^{r} e+\bar{R}_{e}^{r} e=B_{d}^{0}(\tilde{\Phi}+w)-K_{1} e .
$$

Again a candidate function is proposed to find $w$, this function is:

$$
V=\frac{1}{2} e^{\top} D_{e}^{r} e .
$$

It was shown before that $V$ is positive definite. The derivative of the candidate function is:

$$
\dot{V}=e^{\top} D_{e}^{r} \dot{e}
$$

The term $D_{e}^{r} \dot{e}$ in equation (63) is replaced in the last equation, and this results in:

$$
\dot{V}=-e^{\top} C_{e}^{r} e-e^{\top}\left[\bar{R}_{e}^{r}+K_{1}\right] e+e^{\top} B_{d}^{0}(\tilde{\Phi}+w) .
$$

As mentioned before $-e^{\top} C_{e}^{r} e=0$, also, it was shown that $e^{\top}\left[\bar{R}_{e}^{r}+K_{1}\right] e$ is a positive definite function. Now it only remains to show that $e^{\top} B_{d}^{0}(\tilde{\Phi}+w)$ is a negative definite function or a bounded positive definite function. For this demostration is proposed that:

$$
w=-\frac{\rho_{1}^{2}\left(B_{d}^{0}\right)^{\top} e}{\rho_{1}\left\|e^{\top} B_{d}^{0}\right\|+\epsilon} .
$$

Then

$$
\begin{aligned}
e^{\top} B_{d}^{0}(\tilde{\Phi}+w) & =e^{\top} B_{d}^{0}\left(\tilde{\Phi}-\frac{\rho_{1}^{2}\left(B_{d}^{0}\right)^{\top} e}{\rho_{1}\left\|e^{\top} B_{d}^{0}\right\|+\epsilon}\right) \\
& \leq\left\|e^{\top} B_{d}^{0}\right\|\left(\|\tilde{\Phi}\|-\frac{\rho_{1}^{2}\left\|\left(B_{d}^{0}\right)^{\top} e\right\|}{\rho_{1}\left\|e^{\top} B_{d}^{0}\right\|+\epsilon}\right) \\
& \leq\left\|e^{\top} B_{d}^{0}\right\|\left(\frac{\rho_{1}^{2}\left\|e^{\top} B_{d}^{0}\right\|-\rho_{1}^{2}\left\|\left(B_{d}^{0}\right)^{\top} e\right\|+\rho_{1} \epsilon}{\rho_{1}\left\|e^{\top} B_{d}^{0}\right\|+\epsilon}\right) \\
& =\frac{\rho_{1}\left\|e^{\top} B_{d}^{0}\right\| \epsilon}{\rho_{1}\left\|e^{\top} B_{d}^{0}\right\|+\epsilon} \\
& \leq \epsilon .
\end{aligned}
$$

Therefore, $\dot{V}$ is:

$$
\dot{V} \leq-e^{\top}\left[\bar{R}_{e}^{r}+K_{1}\right] e+\epsilon,
$$

and it can say that the robust control law (59) ensures a bounded error of speed and currents [9].

\section{Simulation}

The AC brushless motor parameters (B26S model, HDT manufacturer), sine PWM parameters and EV parameters are listed in the table (I). The desired speed $\dot{q}_{m d}$ is generated by a Bézier polynomials taking into account a simple driving cycle, acceleration $\ddot{q}_{m d}$ and acceleration derivative $\dddot{q}_{m d}$ are generated by a derivation and double derivation of the desired speed, respectively, using MATLAB/Simulink derivation block.

In the simulation a state filter is used to obtain the derivative of $\tau_{L}$. This filter has a state representation as:

$$
\begin{aligned}
& \dot{x}_{1}=x_{2}, \\
& \dot{x}_{2}=-(2 \pi f)^{2} x_{1}-2^{2 / 3} \pi f x_{2}+(2 \pi f)^{2} \tau_{L},
\end{aligned}
$$


TABLE I

SIMULATION PARAMETERS

\begin{tabular}{|c|c|c|c|}
\hline Parameters & Magnitude & Parameters & Magnitude \\
\hline$f_{s}$ & $5 \mathrm{KHz}$. & $r_{e}$ & $0.121 \Omega$ \\
$v_{i}$ & $600 \mathrm{~V}$. & $L_{l s}$ & $1 \times 10^{-5} \mathrm{H}$. \\
$\lambda_{m}$ & $0.262 \mathrm{Vs} / \mathrm{rad}$. & $\bar{L}_{m}$ & $8 \times 10^{-4} \mathrm{H}$. \\
$R_{m}$ & $0.00001 \mathrm{Nms} / \mathrm{rad}$. & $L_{\Delta m}$ & $0 \mathrm{H}$. \\
$n_{p}$ & 4 & $D_{m}$ & $0.022 \mathrm{Kgm}^{2}$. \\
$m$ & $1366 \mathrm{Kg}$ & $\mu_{r r}$ & 0.015 \\
$g$ & $9.8 \mathrm{~m} / \mathrm{s}^{2}$ & $\rho$ & $1.25 \mathrm{Kg} / \mathrm{m}^{3}$ \\
$A$ & $2.66 \mathrm{~m}^{2}$ & $C_{d}$ & 0.23 \\
$\psi$ & $0 \mathrm{rad}$ & $G$ & 5.5 \\
$r$ & $0.2876 \mathrm{~m}$ & $\eta_{g}$ & 0.95 \\
\hline
\end{tabular}

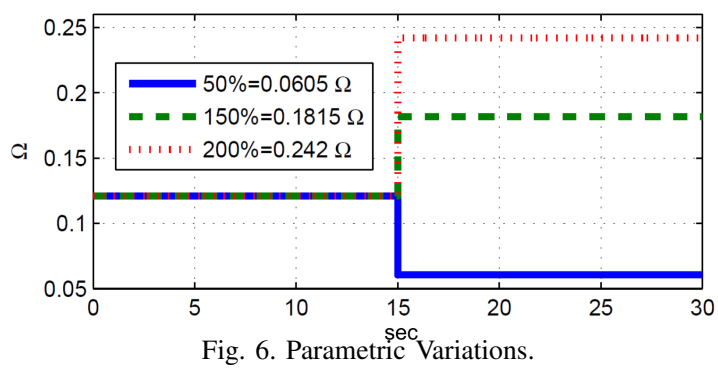

with $f=45 \mathrm{~Hz}$. The filter input is the load torque given by (34) and the filter output are the states $x_{1}$ and $x_{2}$ which are the load torque and its derivative respectively. This outputs are used in equations (33) with the intention of saving computational efforts. The nominal controller gains are $\Gamma_{m}=9000$ and $k=20$. For the robust controller, the gains are also $\Gamma_{m}=9000$ and $k=20$ plus $\rho_{1}=0.121$ and $\epsilon=0.01$.

To probe the robust controller, in the simulations abrupt changes in the winding resistances were considered to evaluate the performance of nominal and robust controllers under unbalanced operating conditions. It is assumed single phase variation in the resistance value. The figure 6 shows the variation in the nominal value of the resistance in $t=15 \mathrm{sec}$. Changes of 50\%, $150 \%$ and $200 \%$ are considered.

On the other hand, to evaluate the controllers performance beyond the variations on the stator resistances, changes in $\psi$ were considered. This variable represents the angle of the surface in which the vehicle moves and is related directly to mechanical parameters like the mass $m$ of the car and gravity $g$. The figure 7 shows the variation in $\psi$, it is clear that until the instant $t=23 \mathrm{sec}$ the car moves over a zero-slope surface, then the next $5 \mathrm{sec}$ the vehicle moves over a road with angle $\psi$ different of zero (ramp-road). At the instant $t=28 \mathrm{sec}$ the ramp road is no more, so the angle $\psi$ returns to zero.

\section{RESUlTs}

\section{A. Under Resistances Variations}

Several simulations were performed, the table II shows the performance indices(Integral of Absolute Error IAE and the Integral of Time multiplied by Absolute Error ITAE) of the nominal and robust controllers for speed tracking under variations in single phase resistance.

In speed control performance, nominal controller shows a lower indices than robust. Here is necessary to say that robust control design was made to deal with parametric uncertainty in stator resistance, this involves the electrical subsistem directly.

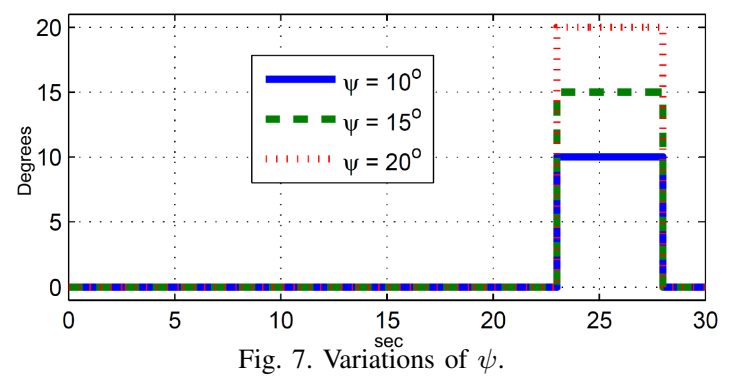

TABLE II

NONLINEAR CONTROLLERS PERFORMANCE

\begin{tabular}{|c|c|c|c|c|}
\hline Controller & Resistance & Variation & IAE & ITAE \\
\hline Nominal & $r_{a}, r_{b}, r_{c}$ & $50 \%$ & 1.132 & 17.63 \\
Robust & $r_{a}, r_{b}, r_{c}$ & $50 \%$ & 1.17 & 18.26 \\
\hline Nominal & $r_{a}, r_{b}, r_{c}$ & $150 \%$ & 1.133 & 17.66 \\
Robust & $r_{a}, r_{b}, r_{c}$ & $150 \%$ & 1.175 & 18.36 \\
\hline Nominal & $r_{a}, r_{b}, r_{c}$ & $200 \%$ & 1.133 & 17.65 \\
Robust & $r_{a}, r_{b}, r_{c}$ & $200 \%$ & 1.171 & 18.27 \\
\hline
\end{tabular}

The next figures shows the results of both nominal and robust controllers under a $200 \%$ variation in $r_{c}$ in $t=15 \mathrm{sec}$. The desired, nominal and robust speed trajectories are shown in figure 8. The maximum amplitude of desired trajectory is $265.6 \mathrm{rad} / \mathrm{sec}=50 \mathrm{Km} / \mathrm{hr}$. It is clearly that a $200 \%$ variation in $r_{c}$ do not affect the speed tracking. Figure 9 shows the electromagnetic torque tracking for both cases, the nominal case presents bigger maximum in $t=27 \mathrm{sec}$ than the robust case. The advantage of robust control over the nominal is visible in the regulation of $\dot{q}_{2}^{r}$ and $\dot{q}_{3}^{r}$, this currents are desired equal to zero. Robust case presents lower currents than nominal case (figures 10 and 11). Lower amplitude currents extend the motor life-time because the heating is less. The table III clearly indicates that robust controller has lower indices for currents regulation than nominal controller.

\section{B. Under Changes on $\psi$}

The table IV shows the performance indices (Integral of Absolute Error IAE and the Integral of Time multiplied by Absolute Error ITAE) of the nominal and robust controllers for speed tracking under variations in the angle $\psi$. Also in this table, it is clearly that in speed tracking the nominal controller is slightly better than robust controller but, this slightly difference is not significant, we have to remember that EV's speed control application does not need a high level of accuracy. It is important to say now that we are interested to reduce the currents amplitude under resistance uncertainty and therefore extend the motor lifetime. The figure 12 shows the speed error for both controllers under a $20^{\circ}$ variation in $\psi$. The amplitude of this error signals is short compared with the reference in figure 8 . The both nominal and robust mechanical subsystems controllers are designed in the same manner, so there is no visible difference between these two signals. Because in this case resistance variations equals to zero, both nominal and robust produces the same currents in the motor stator. The figure 13 shows the current $i_{a}$ produced due to both controllers, also it is clearly that in the instant $t=\left[\begin{array}{ll}23 & 28\end{array}\right] \mathrm{sec}$ the current increased because $\psi$ changed. 
TABLE III

ITAE FOR $i_{d}$ AND $i_{0}$ REGULATION

\begin{tabular}{|c|c|c|}
\hline Controller/current & $i_{d}$ & $i_{0}$ \\
\hline Nominal & 2017 & 2643 \\
Robust & 1719 & 2107 \\
\hline
\end{tabular}

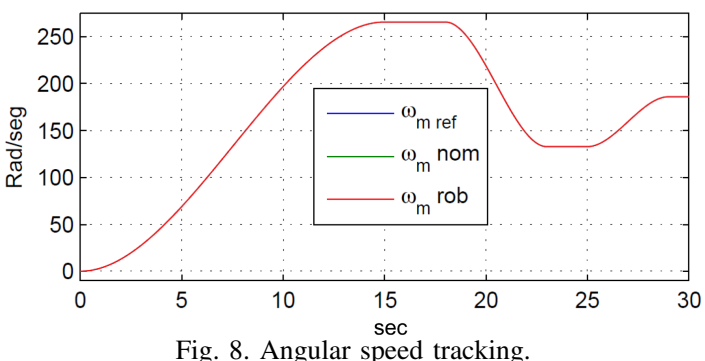

TABLE IV

Nonlinear CONTROLleRs PERFORMANCE Under VARIATIONS IN $\psi$

\begin{tabular}{|c|c|c|c|}
\hline Controller & Angle variation & IAE & ITAE \\
\hline Nominal & $\psi=10^{\circ}$ & 1.147 & 17.62 \\
Robust & $\psi=10^{\circ}$ & 1.148 & 17.65 \\
\hline Nominal & $\psi=15^{\circ}$ & 1.132 & 17.29 \\
Robust & $\psi=15^{\circ}$ & 1.134 & 17.27 \\
\hline Nominal & $\psi=20^{\circ}$ & 1.117 & 16.87 \\
Robust & $\psi=20^{\circ}$ & 1.119 & 16.91 \\
\hline
\end{tabular}

\section{CONCLUSiOnS}

In this paper a EV model is presented. The use of sine PWM technique and power inverter in simulations allows results approximate to reality. An AC brushless motor (also called permanent magnet synchronous motor) model is presented in E-L formulation for two reference frames. Also a mechanical dynamic model for the EV is presented. A robust nonlinear control for AC brusless motors considering parameter uncertainty was presented. The Lyapunov redesign technique was used to obtain an additional feedback signal that is added to the nominal control law to guarantee the uniform ultimate stability in the presence of uncertainties. The parameters of the AC BM need not be precisely known a priori, only the uncertainty bound $\rho_{1}$ is needed to derive the compensation signals. The proposed control law is able to deal with the uncertainties in the winding resistances. The simulation results show that a good tracking behavior is obtained.

\section{ACKNOWLEDGMENT}

The authors wish to thank the anonymous reviewers for their helpful comments and suggestions. This work was supported by CONACYT, México.

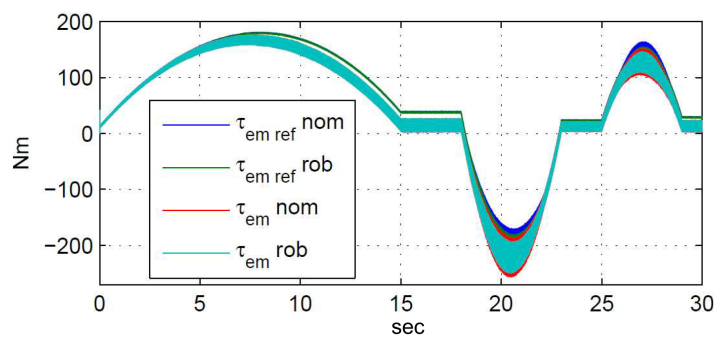

Fig. 9. Electromagnetic torque tracking.

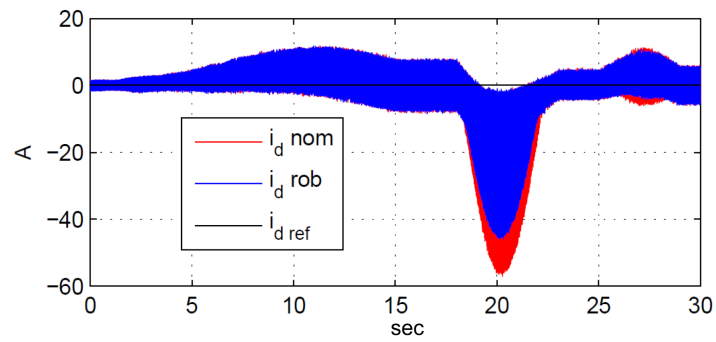

Fig. 10. $i_{d}=\dot{q}_{2}^{r}$ current regulation.

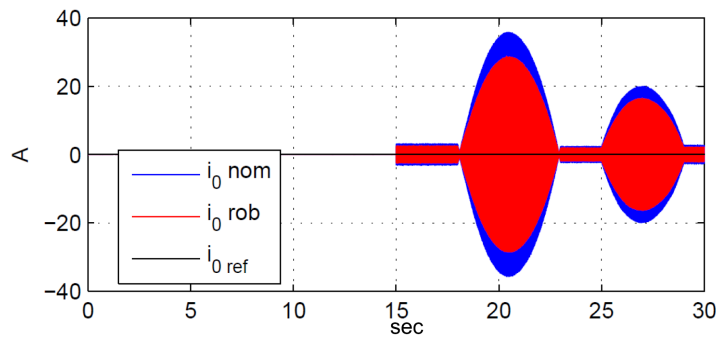

Fig. 11. $i_{q}=\dot{q}_{3}^{r}$ current regulation.

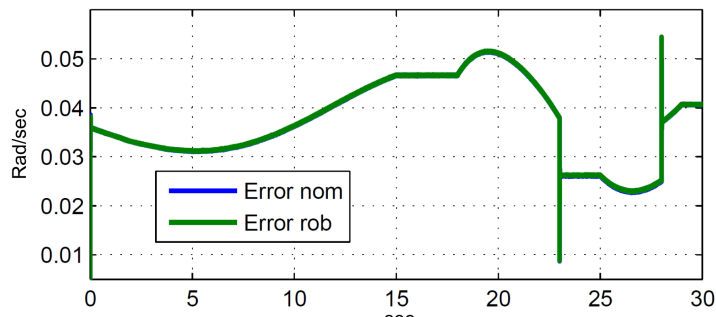

Fig. 12. Speed error for both nominal and robust controllers.

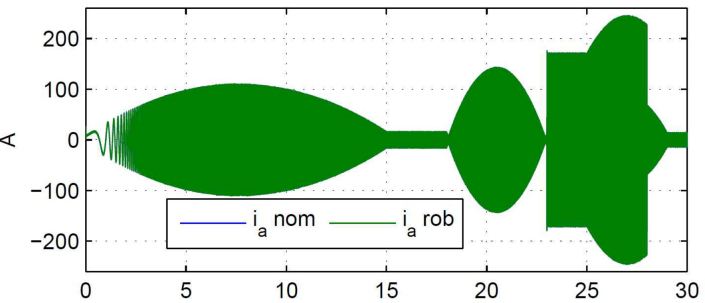

Fig. 13. Current $i_{a}$ for both cases nocminal and robust controllers

\section{REFERENCES}

[1] K.-Y. Cho, "Sensorless control for a PM synchronous motor in a single piston rotary compressor," Journal of Power Electronics, Vol. 6, No. 1, pp. 11-19, Jan. 2006.

[2] J. M. A. Scherpen, D. Jeltsema, and R. Ortega, "An energy-balancing perspective of interconnection and damping assignment control of nonlinear systems," Automatica, Vol. 40, pp. 1643-1646, Sep. 2004.

[3] A. E. Fitzgerald, C. Kingsley, and S. D. Umans, Electric Machinery, McGraw Hill, 2003.

[4] G. V. Guerrero-Ramírez, "Control de Manipuladores de Robots Accionados por Motores de Inducción,” Ph.D. Thesis, Universidad Nacional Autónoma de México, Feb. 2001.

[5] L. Guzzella and A. Sciarretta, Vehicle Propulsion Systems, Springer, 2007.

[6] A. Haddoun, M. Benbouzid, D. Diallo, R. Abdessemed, J. Ghouli, and K. Srairi, " A loss minimization DTC scheme for EV induction motors," IEEE Trans. Veh. Technol, Vol. 56, No. 1, Jan. 2007.

[7] Y. Hori, "Future vehicle driven by electricity and control research on four-wheel-motored UOT electric March II," IEEE Trans. Ind. Electron. pp. 954-962, Oct. 2004

[8] K.-H. Hyun, "Design of a speed controller for permanent magnet synchronous motor in pure electric vehicle applications," International Conference on Control, Automation and Systems, Oct. 2007.

[9] H. K. Khalil, Nonlinear Systems, Prentice Hall, 2002.

[10] H. Komurcugil, "Steady-state analysis and passivity-based control of single-phase PWM current-source inverters," IEEE Trans. Ind. Electron., Vol. 57, No. 3, pp. 1026-1030, Mar. 2010. 
[11] P. Kundur, Power System Stability and Control, McGraw- Hill,Inc, 1994.

[12] S. E. Lyshevski, Electromechanical Systems, Electric Machines And Applied Mechatronics, CRC press, 1999.

[13] R. Ortega, A. Loria, P. J. Nicklasson, and H. Sira-Ramirez, Passivity Based Control of Euler-Lagrange Systems, Springer, 1998.

[14] V. Petrovic, R. Ortega, and A. M. Stankovic, "Interconnection and damping assignment approach to control of PM synchronous motors," IEEE Trans. Contr. Syst. Technol., Vol. 19, No. 6, pp 811-820, Nov. 2001.

[15] A. D. Rajapakse, A. M. Gole, and P. L. Wilson, "Electromagnetic transients simulation models for accurate representation of switching losses and thermal performance in power electronic systems," IEEE Trans. Power Del., Vol. 20, No. 1, pp 319-327, Jan. 2005.

[16] M. H. Rashid, Power Electronics Handbook, Academic Press, 2001.

[17] A. Astolfi, J. Lee, R. Ortega, L. Praly and K. Nam, "Estimation of rotor position and speed of permanent magnet synchronous motors with guaranteed stability," IEEE Trans. Contr. Syst. Technol., pp 1-14, Apr. 2010.

[18] J. L. Tichenor, S. D. Sudhoff, and J. L. Drewniak, "Behavioral IGBT modeling for predicting high frequency effects in motor drives," IEEE Trans. Power Electron., Vol. 15, No. 2, pp 354-360, Mar. 2000.

[19] A. M. Trzynadlowski, Control of Induction Motors, Academic Press, 2001.

[20] S.-C. Yoon and J.-M. Kim, "Sensorless control of a PMSM at low speeds using high frequency voltage injection," Journal of Power Electronics, Vol. 5, No. 1, pp. 11-19, Jan. 2005.

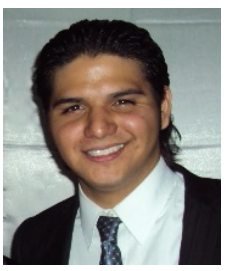

Diego Langarica-Córdoba was born in Veracruz, México. He received the B.Eng. degree in Electronic Engineering from the Technological Institute of Veracruz (with honors), México in 2008, the M.Sc. degree from CENIDET, Morelos, México in 2010. Now, he is pursuing the Ph.D. degree in Engineering at the Laboratoire des Signaux et Systèmes of the Université de Paris Sud XI-SUPELEC, France.

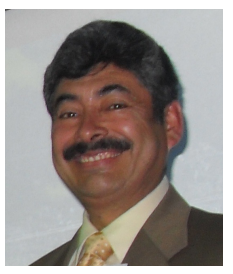

Gerardo V. Guerrero-Ramírez was born in México. He received the professional diploma in electrical engineering from the Instituto Tecnológico de Morelia, México, in 1985, and the M.Sc. degree in Electronic Engineering from the Centro Nacional de Investigación y Desarrollo Tecnológico (CENIDET), Cuernavaca, Morelos, Mexico, in 1994, and the Ph.D. degree in Engineering from the Universidad Nacional Autónoma de México (UNAM), Mexico, in 2001. He is currently professor of department of electronic engineering at the Centro Nacional de Investigación y Desarrollo Tecnológico (CENIDET).

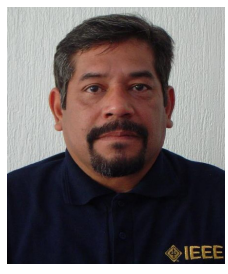

Abraham Claudio-Sánchez was born in Valle Hermoso, Tamps, México. He has obtained his B.Sc. degree and M.Sc. degree in Electrical Engineering from the Instituto Tecnologico de la Laguna, Mexico in 1983 and 1987 respectively. The Diplome d'Etudes Avancées (DEA) and the PhD degree in Electrical Engineering (Power Electronics) from the Institut National Politéchnique de Grenoble (INPG), France in 1992 and 1995 respectively. He realized a Post doctorate research at the Center for Power Electronics Systems-CPES from the Virginia Polytechnic Institute and State University in 2002. Since 1987, he has been with the National Center for Research and Technological Development (CENIDET), Cuernavaca, Mexico as a full time professor in the Power Electronics Group. His field of major interest and experience is characterization and modeling of power semiconductor devices, and he has affinity with other related topics like $\mathrm{AC}$ motor control and fault detection.

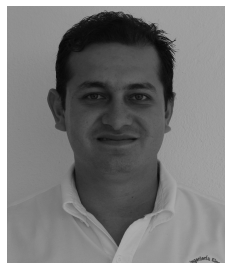

Miguel A. Durán-Fonseca was born in Guanajuato, México, in 1978. He received the B.S. degree in communications and electronic engineering from the University of Colima, México, in 2001, the M.S. degree in electronic engineering from CENIDET, México, in 2004. Since 2004, he has been with the Faculty of Electromechanical Engineering, University of Colima, where he is currently a Research-Professor. Since 2008 he has been studying the Ph.D. degree in electronic engineering in CENIDET, México. His research interest is control of electrical machines applied to electric vehicles.

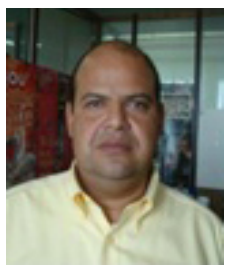

Manuel Adam-Medina was born in Minatitlán, Ver., México. He received the professional diploma in electronic engineering of intrumentation from the Instituto Tecnológico de Minatitlán, México, in 1991, and the M.Sc. degree in electronic engineering from the Centro Nacional de Investigación y Desarrollo Tecnológico (CENIDET), Cuernavaca, Morelos, México, in 1995, and the Ph.D. degree in engineering from the Université Henri Poincaré, Nancy I, France, in 2004. He is currently professor of department of electronic engineering at the Centro Nacional de Investigación y Desarrollo Tecnológico (CENIDET). Since september 1994 he has held a teaching and research position at the Cenidet. His research interests include the multi-model approachs, observers, fault diagnosis, and fault tolerant control systems, with applications to process engineering.

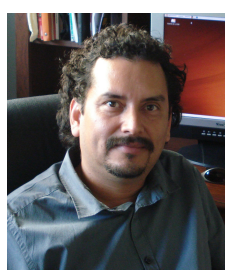

Carlos-Manuel Astorga-Zaragoza was born in Minatitlán, Veracruz, México, in 1967. He received the B.Sc. degree in Electronics and Instrumentation Engineering from the Instituto Tecnológico de Minatitlán, México, in 1992, the M.Sc. degree in Electronics Engineering from the Centro Nacional de Investigación y Desarrollo Tecnológico (CENIDET), Cuernavaca, Morelos, México, in 1993, and the Ph.D. degree in Process Engineering from the Université Claude Bernard Lyon 1, France, in 2001. He was a visiting researcher at the Centre de Recherche en Automatique de Nancy, France, from January to December 2009. Since 1993, he has held teaching and research positions at the CENIDET. His research interests include the nonlinear observers, fault diagnosis, and control systems with applications, in particular to process engineering. 